\title{
Evaluation of the Quality of Cardiovascular Surgery Care Using Risk Stratification Analysis According to the EuroSCORE Additive Model
}

\author{
Yoshito Kawachi, MD; Atsuhiro Nakashima, MD; Yoshihiro Toshima, MD; \\ Kouich Arinaga, MD; Hiroshi Kawano, MD
}

\begin{abstract}
Risk stratification according to the EuroSCORE additive model of 803 consecutive patients undergoing heart and thoracic aorta surgery using cardiopulmonary bypass from August 1994 to December 2000 was performed. The population was divided into 5 clinically relevant risk categories: $0-2 \%$ risk, 3-5\% risk, $6-8 \%$ risk, $9-11 \%$ risk, and $12+\%$ risk. Observed and predicted mortalities were compared within 3 groups of patients divided by year of operation (early: August 1994 to September 1996, n=260; middle: October 1996 to September 1998, $\mathrm{n}=259$; late: October 1998 to December $2000, \mathrm{n}=284$ ). Overall hospital mortality was $4.5 \%$; predicted mortality was $5.3 \%$ in the early, $5.1 \%$ in the middle, and $5.4 \%$ in the late period; observed mortality was $6.5 \%, 3.9 \%$, and $3.2 \%$, respectively ( $\mathrm{p}=0.0024$ in early vs late). In the early period, observed mortality was lower than predicted mortality in the $0-2 \%$ and $3-5 \%$ risk categories, but higher in the other categories. Moreover, observed mortality increased markedly with the increase in predicted mortality. In the late period, observed mortality was lower than predicted mortality in all 5 risk categories. The EuroSCORE is clinically relevant index for constructing a risk stratification scoring system for Japanese cardiovascular patients as well and shows that the quality of surgical care has improved gradually over the years. (Circ J 2002; 66: 145-148)
\end{abstract}

Key Words: Cardiovascular surgery; EuroSCORE; Observed mortality; Predicted mortality; Risk stratification

$\mathbf{F}$ or an evaluation of the results of cardiovascular surgery, crude operative mortality rate (ie, the number of deaths divided by the number of operations) is generally employed, although these raw outcome data do not reflect the preoperative condition of the individual patient. Therefore, to resolve that problem, risk stratification analysis was developed ${ }^{1-3}$ for 2 major uses: one is estimating the risk of surgical mortality faced by an individual patient, as an aid to patients and physicians contemplating cardiovscular surgery, and the other is as a tool for measuring the quality of surgical care. Recently, the European System for Cardiac Operative Risk Evaluation (Euro SCORE) additive model was introduced, and it has global scores with simple and objective definitions provided at the data collection stage? We used the EuroSCORE algorithm to assess the biennial trend towards a reduction in operative mortality rate in order to assess the quality of cardiac and thoracic aortic surgical care in our institution.

\section{Methods}

Between August 1994 and December 2000, 803 consecutive patients had surgery for cardiac and thoracic aortic disease: 486 men $(61 \%)$ and 317 women (39\%), aged from 13 to 90 years old (mean, $63 \pm 12$ years). All patients selected had to have undergone cardiovascular surgery with cardio-

(Received September 12, 2001; revised manuscript received October 31, 2001; accepted November 13, 2001)

Cardiovascular Surgery, Clinical Research Institute, National Kyushu Medical Center Hospital, Fukuoka, Japan

Mailing address: Yoshito Kawachi, MD, National Kyushu Medical Center Hospital, 1-8-1 Jigyo-hama, Chuo-ku, Fukuoka 810-8563,

Japan. E-mail: kawachiy@qmed.hosp.go.jp pulmonary bypass, so those who had off-pump coronary artery bypass grafting (CABG) were excluded. The patient population was divided into 3 time periods: early (August 1994 through September 1996, n=260), middle (October 1996 through September 1998, n=259), and late (October 1998 through December 2000, $\mathrm{n}=284$ ). The distribution of the surgery performed is shown in Table 1 , and the data for the EuroSCORE risk factors, which was entered into a database, is shown in Table2. Expected or predicted mortality was calculated for individual patients using the EuroSCORE additive model, arranged sequentially in order of predicted score. The study population was divided into 5 clinically relevant risk categories: $0-2 \%$ risk, $3-5 \%$ risk, 6-8\% risk, 9-11\% risk, and $12+\%$ risk. Mortality was defined as death from any cause within 30 days of operation or within the same hospital admission, and expected mortality was compared with observed or actual mortality for each risk category.

The continuous data were expressed as the mean \pm one standard deviation, and categorical variables were expressed as percentages. Statistical analysis was conducted using StatView 5.0 (SAS Institute Inc, Cary, NC, USA). Comparison of 2 groups was performed for categorical variables with the chi-square test with $2 \times 2$ contingency tables or Fisher's exact test as appropriate. The receiver operating characteristic (ROC) curves were plotted to assess the discrimination abilities (accuracy) of the EuroSCORE4 The area under the ROC curve was calculated as an index for how well the model could discriminate between patients who lived and those who died. The discriminative power of the model is thought excellent if the area under the ROC curve is $>0.8$, very good if $\geq 0.8>0.75$, and good if $\geq 0.75$ $>0.70$. Variables were judged significant at $\mathrm{p}<0.05$. 
Table 1 Distribution of the Surgical Procedures

\begin{tabular}{|c|c|c|c|c|c|}
\hline \multirow{2}{*}{ Procedure } & \multicolumn{4}{|c|}{ No. of patients } & \multirow{2}{*}{$\begin{array}{c}\text { Mortality } \\
\text { total }\end{array}$} \\
\hline & '94.8-'96.9 & '96.10-'98.9 & '98.10-'00.12 & Total & \\
\hline CABG only & $94(36 \%)$ & $90(35 \%)$ & $117(41 \%)$ & $301(38 \%)$ & $12(4.0 \%)$ \\
\hline$C A B G+$ Valve & $10(3.8 \%)$ & $11(4.2 \%)$ & $7(2.5 \%)$ & $28(3.5 \%)$ & $3(11 \%)$ \\
\hline Valve only & $85(33 \%)$ & $89(34 \%)$ & $82(29 \%)$ & $256(32 \%)$ & $4(1.6 \%)$ \\
\hline TAA only & $36(14 \%)$ & $41(16 \%)$ & $44(15 \%)$ & $121(15 \%)$ & $14(12 \%)$ \\
\hline TAA + Others & $4(1.5 \%)$ & $3(1.2 \%)$ & $7(2.5 \%)$ & $14(1.7 \%)$ & $2(14 \%)$ \\
\hline MI complication & $2(0.8 \%)$ & $4(1.5 \%)$ & $0(0 \%)$ & $6(0.7 \%)$ & $1(17 \%)$ \\
\hline Others & $29(11 \%)$ & $21(8.1 \%)$ & $27(9.5 \%)$ & $77(9.6 \%)$ & $0(0 \%)$ \\
\hline Total & $260(100 \%)$ & $259(100 \%)$ & $284(100 \%)$ & $803(100 \%)$ & $36(4.5 \%)$ \\
\hline
\end{tabular}

$C A B G$, coronary artery bypass grafting; TAA, thoracic aortic aneurysm; $M I$, myocardial infarction.

Table 2 Results of Univariate Analysis Regarding the Risk Factors of the EuroSCORE

\begin{tabular}{|c|c|c|c|c|c|}
\hline \multirow{2}{*}{ Risk factor } & \multicolumn{2}{|c|}{ With risk factor } & \multicolumn{2}{|c|}{ Without risk factor } & \multirow{2}{*}{$p$ value } \\
\hline & Total pts & Mortality (\%) & Total pts & Mortality (\%) & \\
\hline \multicolumn{6}{|l|}{ Age } \\
\hline $60-64$ & 137 & 2.2 & 237 & 4.6 & 0.85 \\
\hline $65-69$ & 174 & 4.0 & & & \\
\hline $70-74$ & 148 & 4.1 & & & \\
\hline $75-79$ & 78 & 7.7 & & & \\
\hline $80-84$ & 25 & 12 & & & \\
\hline $85-89$ & 3 & 0 & & & \\
\hline$\geq 90$ & 1 & 0 & & & \\
\hline Female gender & 315 & 3.2 & 488 & 5.3 & 0.17 \\
\hline Chronic pulmonary disease & 8 & 0 & 795 & 4.5 & $>0.9999$ \\
\hline Extracardiac arteriopathy & 66 & 4.5 & 737 & 4.5 & $>0.9999$ \\
\hline Neurological dysfunction & 16 & 13 & 787 & 4.3 & 0.16 \\
\hline Previous cardiac surgery & 53 & 7.5 & 750 & 4.3 & 0.29 \\
\hline Serum creatinine $>2 \mathrm{mg} / \mathrm{dl}$ & 32 & 13 & 771 & 4.2 & 0.050 \\
\hline Active endocarditis & 15 & 6.7 & 788 & 4.4 & 0.50 \\
\hline Critical preoperative state & 59 & 25 & 744 & 2.8 & $<0.0001$ \\
\hline Unstable angina & 71 & 13 & 732 & 3.7 & 0.003 \\
\hline \multicolumn{6}{|l|}{$L V E F$} \\
\hline $30-50 \%$ & 102 & 6.9 & 686 & 3.6 & 0.013 \\
\hline$<30 \%$ & 15 & 27 & & & \\
\hline Recent myocardial infarct & 63 & 16 & 740 & 3.5 & 0.0002 \\
\hline Pulmonary hypertension & 19 & 21 & 784 & 4.1 & 0.008 \\
\hline Emergency & 110 & 16 & 693 & 2.6 & $<0.0001$ \\
\hline Other than isolated $C A B G$ & 406 & 3.0 & 397 & 6.0 & 0.040 \\
\hline Surgery on thoracic aorta & 134 & 12 & 669 & 3.0 & $<0.0001$ \\
\hline Postinfarct septal rupture & 3 & 33 & 800 & 4.4 & 0.13 \\
\hline
\end{tabular}

$L V E F$, left ventricular ejection fraction; $C A B G$, coronary artery bypass grafting.

\section{Results}

Overall hospital mortality was $4.5 \%$ (36 of 803 patients); the mortality rate for each surgical procedure is shown in Table 1 and Table 2 shows the impact of the EuroSCORE risk factors on hospital mortality based on univariate analysis. Eight of the 17 variables influenced mortality significantly; namely serum creatinine $>2 \mathrm{mg} / \mathrm{dl}$, critical preoperative state (ventricular tachycardia or fibrillation or aborted sudden death, preoperative cardiac massage, preoperative ventilation before arrival in the anaesthetic room, preoperative inotropic support, intraaortic balloon counterpulsation or preoperative acute renal failure), unstable angina (rest angina requiring intravenous nitrates until arrival in the anaesthetic room), left ventricular dysfunction (left ventricular ejection fraction $<50 \%)$, recent myocardial infarct $(<90$ days), pulmonary hypertension (systolic pulmonary artery pressure $>60 \mathrm{mmHg}$ ), emergency (carried out on referral before the beginning of the next working day), and surgery on the thoracic aorta. Any procedure other than isolated
CABG (major cardiac procedure other than or in addition to $\mathrm{CABG}$ ) was associated with decreased risk.

Fig 1 shows patient distribution by risk classification: the percentage of patients with a EuroSCORE value of $6 \%$ or higher was 39\% in the early, 35\% in the middle, and $43 \%$ in the late period, an increase in the late period without significance ( $\mathrm{p}=0.079$ in middle vs late). Observed mortality gradually decreased with the years (Fig 2 ). In the early period, observed mortality was lower than predicted mortality in the $0-2 \%$ and $3-5 \%$ risk categories but higher in the other categories (Fig 3). Moreover, observed mortality increased markedly with an increase in predicted mortality. In the late period, observed mortality was lower than predicted mortality in all risk categories (Fig 4): predicted overall mortality was $5.3 \%$ in the early, $5.1 \%$ in the middle, and $5.4 \%$ in the late period and observed overall mortality was $6.5 \%, 3.9 \%$, and $3.2 \%$, respectively ( $\mathrm{p}=0.0024$ in early vs late). The area under the ROC curve was 0.82 overall, 0.83 in the early and middle periods, and 0.81 in the late period (Fig 5). 


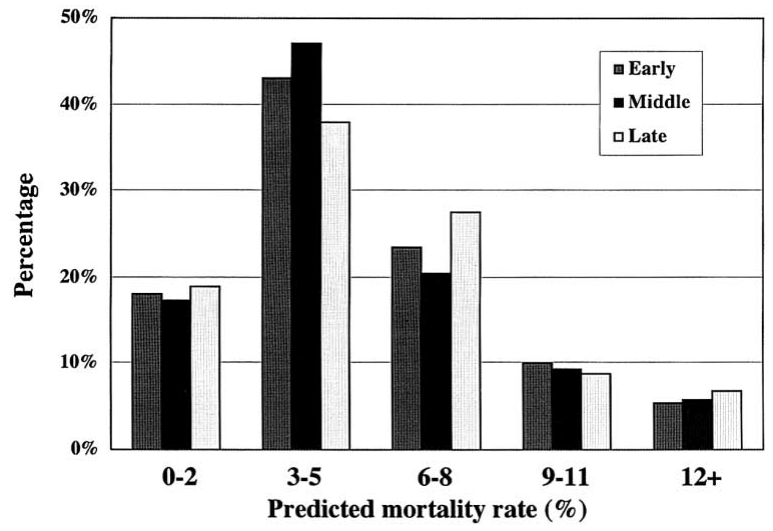

Fig 1. Patient distribution according to risk stratification in each period.

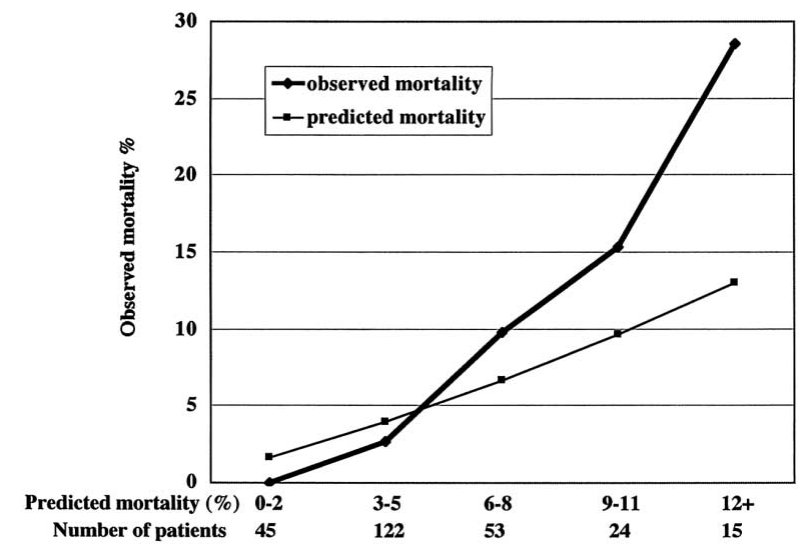

Fig 3. Comparison between predicted mortality and observed mortality in the early period.

\section{Discussion}

Being able to compare surgical outcome at different times and at different institutions is the major advantage of risk stratification because evaluation of the surgical mortality rates of hospitals and surgeons can only be fair and realistic when the observed mortality rates are compared with predicted mortality rates with preoperative risk factors taken into account 6 Risk stratification has become an important tool in assessing the quality of surgical care for the increasing age, disease severity, and comorbidity of patients undergoing heart and thoracic aorta surgery. Quality monitoring is a mainstay of good surgical practice and since August 1994 when the hospital opened, we have used the Parsonnet additive mode $^{3}$ because of its classic and easy application? but have found it difficult to predict the surgical mortality of thoracic aortic surgery with this algorithm? In 1999, Nashef et al introduced EuroSCORE, in which the risk factor of surgery on thoracic aorta was associated with an increased mortality at a score of 3 ? Moreover, it had global scores and simple and objective definitions at the data collection stage. EuroSCORE consists of 3 groups of weighted risk factors (additive \% predicted mortality). Patient-related factors are age $>60$ ( 1 point per 5 years or part thereof), female gender (1), chronic pulmonary disease (1), extracardiac arteriopathy (2), neurological dysfunction (2), previous cardiac surgery (3), serum creatinine $>2 \mathrm{mg} / \mathrm{dl}$ (2), active endocarditis (3) and critical preoperative state (3). Cardiac factors are

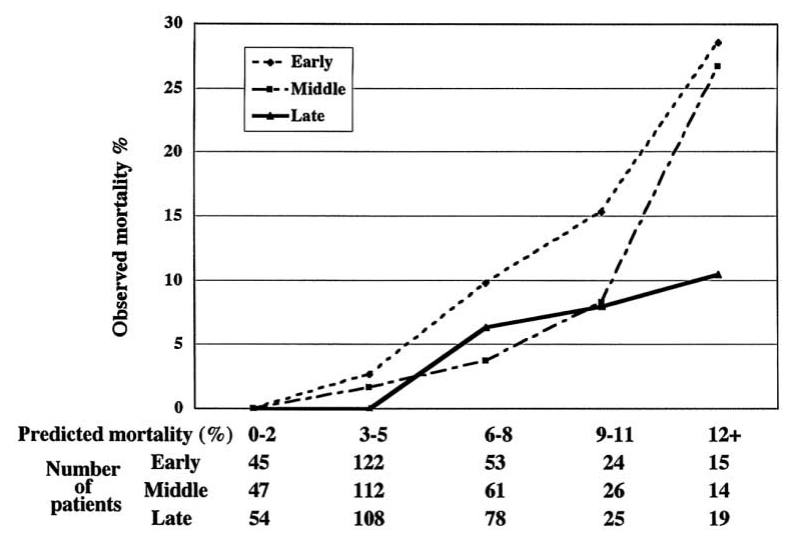

Fig 2. Observed mortality according to risk stratification analysis in each period.

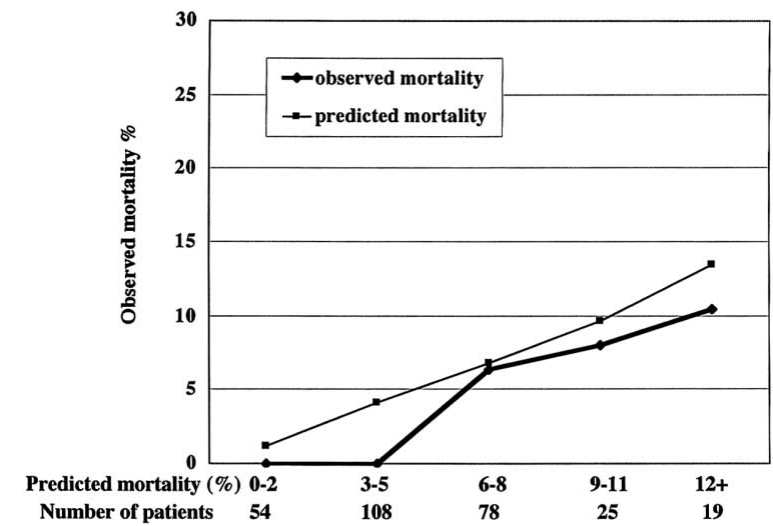

Fig 4. Comparison between predicted mortality and observed mortality in the late period.

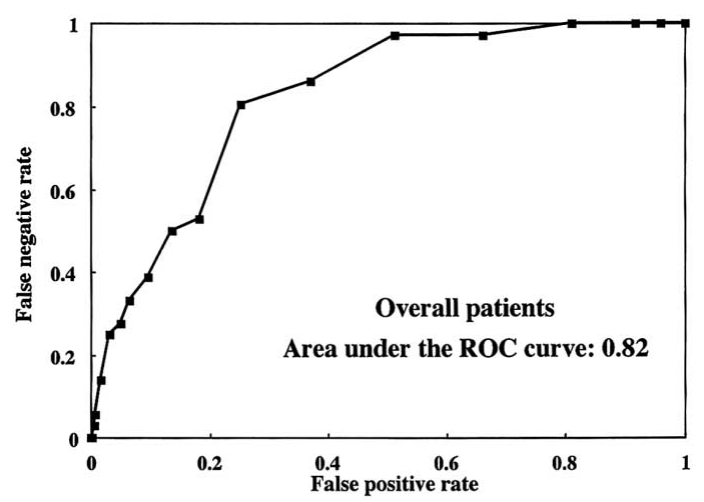

Fig 5. The receiver operating characteristic (ROC) curve in all patients.

unstable angina on intravenous nitrates (2), reduced left ventricular ejection fraction (30-50\%: 1, <30\%: 3), recent ( $<90$ days) myocardial infarction (2) and pulmonary systolic pressure $>60 \mathrm{mmHg}$ (2). Operation-related factors are emergency (2), other than isolated CABG (2), thoracic aorta surgery (3) and surgery for postinfarct septal rupture (4) (Table 2)? The predicted mortality rate of individual patients is the sum of these appropriate scores.

The risk stratification analysis allows a comparison of the case mix, most readily represented by the mean expected 
Table 3 Comparison of the Surgical Results

\begin{tabular}{|c|c|c|c|c|}
\hline & \multicolumn{3}{|c|}{ EuroSCORE } & \multirow{2}{*}{ Total } \\
\hline & $0-2$ & $3-5$ & $6+$ & \\
\hline \multicolumn{5}{|l|}{ Nashef et $a l^{2}$} \\
\hline Patients & 4,529 & 5,977 & 4,293 & 14,799 \\
\hline Percentage (\%) & 31 & 40 & 29 & 100 \\
\hline Mortality (\%) & 0.8 & 3.0 & 11 & 4.7 \\
\hline \multicolumn{5}{|l|}{ Pitkanen et al $[R]^{10}$} \\
\hline Patients & 714 & 548 & 269 & 1,531 \\
\hline Percentage (\%) & 47 & 36 & 18 & 100 \\
\hline Mortality (\%) & 0.6 & 1.3 & 7.8 & 2.1 \\
\hline \multicolumn{5}{|l|}{ Pitkanen et al $[P]^{10}$} \\
\hline Patients & 326 & 309 & 186 & 821 \\
\hline Percentage (\%) & 40 & 38 & 23 & 100 \\
\hline Mortality (\%) & 0.3 & 0.6 & 3.2 & 1.1 \\
\hline \multicolumn{5}{|l|}{ Present study } \\
\hline Patients & 146 & 342 & 315 & 803 \\
\hline Percentage (\%) & 18 & 43 & 39 & 100 \\
\hline Mortality (\%) & 0 & 1.5 & 9.8 & 4.5 \\
\hline
\end{tabular}

$R$, retrospective validation database; $P$, prospective validation database.

risk or by the percentage of patients whose risk exceeds poor (a EuroSCORE value of $6 \%$ or higher)? In the present patient population, the mean expected mortality rate was $5.3 \%$ in the early, $5.1 \%$ in the middle, and $5.4 \%$ in the late period, and the percentage of patients with a predicted risk $6 \%$ or higher was $39 \%, 35 \%$ and $43 \%$, respectively. From these results, the preoperative and operative risk was slightly higher in patients during the late period without significance. The overall observed mortality decreased markedly from $6.5 \%$ in the early period to $3.2 \%$ in the late period ( $\mathrm{p}=0.0024)$, with the surgical result for patients with a $6 \%$ or higher risk particularly improved (Fig 2). However, it should be mentioned that each risk category of $6 \%$ or higher had a small patient number. From this risk stratification analysis, we believe that the quality of cardiovascular surgery had gradually improved over the course of years. The primary factors for this improvement are under consideration, but may be the conversion of cardioplegia from crystalloid to blood, progress in patient care during the critical preoperative state and training of staff and doctors in theater and the intensive care unit.

Comparable external data is necessary to put individual institutional findings in perspective. The EuroSCORE is suited for this because the data can be collected easily using standardized definitions. Our observed mortality was parallel to the EuroSCORE application data reported by Nashef et al (Table 3)? Pitkanen et al reported good operative results, particularly in the patient population with $6 \%$ or higher risk $!^{10}$ The percentage of the present patients whose predicted risk exceeded $6 \%$ was significantly higher than in other reports $(p<0.0001)$, which means that higher risk patients undergo surgery more frequently in our institution than in other institutions.

In the United States another risk stratification method for cardiac surgery, the Society of Thoracic Surgeons National Database (STS database) has gradually gained authority $1,11,12$ Individual institutions can enter their patient population data into computer software, obtain the predicted mortality rate of each patient and compare their risk-adjusted surgical results with the national standard derived from the North American patient population. However, the computer software for the STS database is expensive whereas Euro SCORE is a free, simple, objective and up-to-date system for assessing cardiac and thoracic aortic surgery?
In conclusion, using to the EuroSCORE additive model we can fairly calculate the predicted risk of surgical mortality faced by an individual patient and evaluate the actual mortality rates of hospitals with risk stratification scoring analysis of the case mix undergoing cardiovascular surgery. The EuroSCORE is a clinically relevant index not only for European but also for Japanese cardiovascular patients. The quality of surgical care in our institution has gradually improved over the years.

\section{References}

1. Hattler BG, Madia C, Johnson C, Armitage JM, Hardesty RL, Kormos L, et al. Risk stratification using The Society of Thoracic Surgeons program. Ann Thorac Surg 1994; 58: 1348-1352.

2. Nashef SAM, Roques F, Michel P, Gauducheau E, Lemeshow S, Salamon R, et al. European system for cardiac operative risk evaluation (EuroSCORE). Eur J Cardiothorac Surg 1999; 16: 9-13.

3. Parsonnet V, Dean D, Bernstein AD. A method of uniform stratification of risk for evaluating the results of surgery in acquired adult heart disease. Circulation 1989; 79(Suppl I): I-3-I-12.

4. Swets JA. Measuring the accuracy of diagnostic systems. Science 1988; 240: 1285-1293.

5. Roques F, Nashef SAM, Michel P, Pintor PP, David M, Baudet E, et al. Does EuroSCORE work in individual European countries? Eur J Cardiothorac Surg 2000; 18: 27-30.

6. Bernstein AD, Parsonnet V. Bedside estimation of risk as an aid for decision-making in cardiac surgery. Ann Thorac Surg 2000; 69: 823 828.

7. Kawachi Y, Nakashima A, Toshima Y, Komesu I, Kimura S, Arinaga K. Risk stratification analysis of operative mortality of coronary artery bypass surgery. Jpn J Thorac Cardiovasc Surg 2001; 49: 557-563.

8. Kawachi Y, Nakashima A, Toshima Y, Arinaga K, Kawano H. Risk stratification analysis of operative mortality in heart and thoracic aorta surgery: Comparison between Parsonnet and EuroSCORE additive model. Eur J Cardiothorac Surg 2001; 20: 961-966.

9. Parsonnet V, Bernstein AD, Gera M. Clinical usefulness of risk-stratified outcome analysis in cardiac surgery in New Jersey. Ann Thorac Surg 1996; 61: S8-S11.

10. Pitkanen O, Niskanen M, Rehnberg S, Hippelainen M, Hynynen M. Intra-institutional prediction of outcome after cardiac surgery: Comparison between a locally derived model and the EuroSCORE. Eur J Cardiothorac Surg 2000; 18: 703-710.

11. Ferguson TB Jr, Dziuban SW Jr, Edwards FH, Eiken MC, Shroyer ALW, Pairolero PC, et al. The STS National Database: Current changes and challenges for the new millennium. Ann Thorac Surg 2000; 69: 680-691.

12. Grover FL. The Society of Thoracic Surgeons National Database: Current status and future directions. Ann Thorac Surg 1999; 68: 367-373. 